\title{
Controle Adaptativo de Semáforo com Pelotões Veiculares Mais Acurados
}

\author{
Arinaldo Lopes da Silva ${ }^{1}$, André Castelo Branco Soares ${ }^{1}$ \\ ${ }^{1}$ Programa de pós-graduação em Ciência da Computação (PPGCC) \\ Universidade Federal do Piauí (UFPI) \\ CEP 64.049-550 - Teresina - PI - Brasil \\ \{arinaldo, andre.soares\}@ufpi.edu.br
}

\begin{abstract}
Traffic congestion in large urban centers has been increasing every year and hampering the mobility of people. The timing configuration of traffic lights is an important factor to be considered. Using vehicular networks, this paper presents an adaptive control algorithm of traffic light reworked from an improvement of the best solution found in the literature so far, the ITLC (Intelligent Traffic Light Controlling). The contributions of this work focus on how the platoons of vehicles are configured and updated. Through simulations in synthetic environment and realistic environment in the city of Bologna in Italy, it was found that the enhanced algorithm showed superior results in terms of throughput of the traffic light and it reduces the average delay per vehicle, the average $\mathrm{CO}_{2}$ emission and signaling overhead compared to the ITLC.
\end{abstract}

Resumo. Os congestionamentos de trânsito nos grandes centros urbanos vêm aumentando a cada ano e prejudicando a mobilidade das pessoas. A configuração de temporização dos semáforos é um fator importante a ser considerado. Utilizando redes veiculares, este trabalho apresenta um algoritmo de controle adaptativo de semáforo reformulado a partir de um aperfeiçoamento da melhor solução encontrada na literatura até então, o ITLC (Intelligent Traffic Light Controlling). As contribuições deste trabalho incidem na forma como os pelotões de veículos são formados e atualizados. Por meio de simulações em ambiente sintético e em ambiente realístico na cidade de Bolonha na Itália, verificou-se que o algoritmo reformulado apresentou resultados superiores em termos de vazão de veículos no semáforo, além de reduzir o atraso médio dos veículos, a emissão média de $\mathrm{CO}_{2}$ e o overhead de sinalização quando comparado ao ITLC.

\section{Introdução}

O crescente número de veículos nos grandes centros urbanos vem aumentando os congestionamentos de trânsito e, consequentemente, prejudicando a mobilidade urbana. Além disso, causam impactos negativos na saúde da população, na economia e no meio ambiente.

Os Sistemas Inteligentes de Transporte (ITS - Intelligent Transportation Systems) têm se apresentado como uma opção para reduzir esses impactos negativos. Além de melhorar a mobilidade urbana, o uso de ITS também contribui indiretamente para a sustentabilidade dos sistemas de transporte, visto que boa parte de suas aplicações reduz as emissões de dióxido de carbono $\left(\mathrm{CO}_{2}\right)$ dos veículos [Lee et al. 2015]. 
As redes veiculares, conhecidas como VANETs (VANETs - Vehicular Ad hoc Networks), são redes que permitem a comunicação entre veículos (comunicação V2V Vehicle-to-Vehicle) e entre veículos e infraestruturas fixas localizadas às margens das vias (comunicação V2I - Vehicle-to-Infrastructure) [Booysen et al. 2011]. Seus nós apresentam alta mobilidade e trajetórias restritas às vias de circulação. As VANETs possibilitam o desenvolvimento de aplicações interessantes para a redução de congestionamentos de trânsito e melhoria na mobilidade em vários pontos das vias urbanas.

Um desses pontos são as interseções entre as vias. A configuração de temporização dos semáforos nas interseções é um fator importante a ser considerado. Em geral, a maioria dos semáforos nas interseções opera de acordo com uma configuração de tempo fixo. Para controlar o fluxo de veículos nas interseções de forma mais eficiente, é preciso que os semáforos operem de forma dinâmica, considerando informações de tráfego em cada uma das vias que ele controla. As redes veiculares são uma forma para obter essas informações de tráfego.

Dentre as soluções que realizam o controle dinâmico de semáforos por meio das redes veiculares, o algoritmo ITLC [Younes and Boukerche 2016] tem se destacado com relação à otimização do controle semafórico em dois aspectos: $(i)$ aumento da vazão de veículos que passam pelo semáforo e (ii) redução do atraso médio dos veículos resultante da interseção sinalizada com semáforo.

O foco deste trabalho é propor um algoritmo aprimorado a partir do ITLC introduzindo melhorias em dois pontos: $(i)$ na forma como ele agrupa os veículos que pretendem passar pela interseção (personalizando esses agrupamentos de acordo com as velocidades individuais de cada fluxo) e (ii) no modo como ele mantém informações atualizadas sobre esses agrupamentos (realizando atualizações não apenas no início, mas também durante o ciclo semafórico).

O restante deste artigo está organizado da seguinte forma. A Seção 2 apresenta alguns trabalhos relacionados. A Seção 3 detalha as melhorias propostas. Os resultados obtidos na avaliação de desempenho são discutidos na Seção 4. Por fim, a Seção 5 apresenta as conclusões e pondera possíveis trabalhos futuros.

\section{Trabalhos Relacionados}

Dentre as soluções que vêm sendo propostas para o controle adaptativo de semáforos, utilizando as redes veiculares, duas formas de programação semafórica podem ser observadas: (i) programação por análise individual de veículo, como a apresentada em [Hsieh and Tsai 2013], e (ii) programação por análise de grupos de veículos, chamados de pelotões. Os seguintes trabalhos utilizam pelotões em suas propostas: [Pandit et al. 2013] [Younes and Boukerche 2016] e [Younes et al. 2016]. De maneira geral, reduzir o atraso médio dos veículos no semáforo e aumentar a vazão de veículos que podem passar pela interseção são os principais objetivos desses trabalhos.

A solução apresentada em [Hsieh and Tsai 2013] implementa um componente de armazenamento (Storage Unit), um analisador de mensagens (Message Analyzer) e um conjunto de regras (Policy Rule) para realizar a programação semafórica. Todos esses elementos de controle são acoplados ao semáforo para tratar as informações enviadas na comunicação V2I. O princípio básico de funcionamento é a atribuição de tempos não 
nulos para cada fase do ciclo semafórico. O tempo de uma fase é decrementado de uma unidade para cada veículo que se aproxima da interseção por uma aproximação com sinal vermelho. Como resultado, há sempre uma priorização do tráfego na via de maior fluxo. Nessa solução, mesmo que não haja tráfego em uma das vias, os veículos da outra via precisam esperar o tempo total ou parcial da fase.

Um mecanismo chamado OAF (Oldest Arrival First) é proposto em [Pandit et al. 2013]. A proposta formula o problema de controle de semáforo como um problema de escalonamento de tarefas em um processador, com as tarefas correspondendo a pelotões de veículos. Partindo da premissa de que as tarefas (pelotões mapeados) são todas de igual tamanho (duração), a proposta então utiliza o algoritmo OJF (Oldest Job First) em tempo real para realizar a programação semafórica. Um pelotão que chega primeiro à interseção recebe o direito de passagem primeiro. Quando chegam mais pelotões, estes são inseridos em uma fila por ordem de chegada. Essa priorização reduz o atraso dos veículos mas não garante que o semáforo trabalhe com a maior vazão possível. Isso porque o tamanho dos pelotões é definido de acordo com o tempo que eles levam para passar pela interseção e não pela quantidade de veículos que eles possuem. O OAF utiliza o mesmo tempo de verde para todos os pelotões, ou seja, não há fases com durações diferentes. Ainda sobre a vazão de veículos, nenhuma avaliação foi realizada a respeito dessa métrica no trabalho.

Em [Younes and Boukerche 2016] é apresentado um algoritmo, chamado ITLC. Ele define uma área virtual no entorno da interseção de modo que os veículos dentro dessa área são considerados como candidatos aptos a passar pela interseção. A Equação 1 define a distância entre o semáforo e o limite da área de aptos $D_{a a}$ com base na velocidade média de todos os $n$ fluxos $V_{f t(i)}$ e o tempo de verde máximo $T_{\text {max }}$, permitido para o semáforo.

$$
D_{a a}=\frac{\sum_{i=1}^{n} V_{f t(i)}}{n} \times T_{\max }
$$

Nessa área de aptos, no máximo um pelotão para cada fluxo é considerado a cada seleção de fase do ciclo semafórico. A coleta de informações sobre os pelotões (densidade do tráfego, velocidade do tráfego e tempo estimado de viagem) é realizada por meio do protocolo ECODE (Efficient COngestion DEtection) [Younes and Boukerche 2015].

O Algoritmo 1 mostra como funciona o ITLC. Inicialmente, as densidades e os tempos estimados de viagem de todos os pelotões são calculados (linha 2). Dois fluxos de tráfego ( $i 1$ e $i 2$ ) são candidatos a passar simultaneamente com o pelotão de mais alta densidade $j$ (linhas 4-5). O fluxo de tráfego que tiver o pelotão de maior densidade entre esses dois fluxos elegíveis é selecionado para passar na mesma fase com o pelotão de mais alta densidade.

Em seguida, assim que dois fluxos de tráfego são incluídos na fase, o maior tempo necessário $\left(T_{p(i)}\right)$ é selecionado para a respectiva fase escalonada (linha 7 ou 10) por meio da função schedule(). O valor de $T_{p(i)}$ é determinado pela Equação 2, onde: $D_{u v(i)}$ é a distância do último veículo do pelotão em relação ao semáforo, $\theta$ é um valor estimado para o tempo de retardo do primeiro veículo do pelotão e $V_{p(i)}$ é a velocidade média do pelotão. 


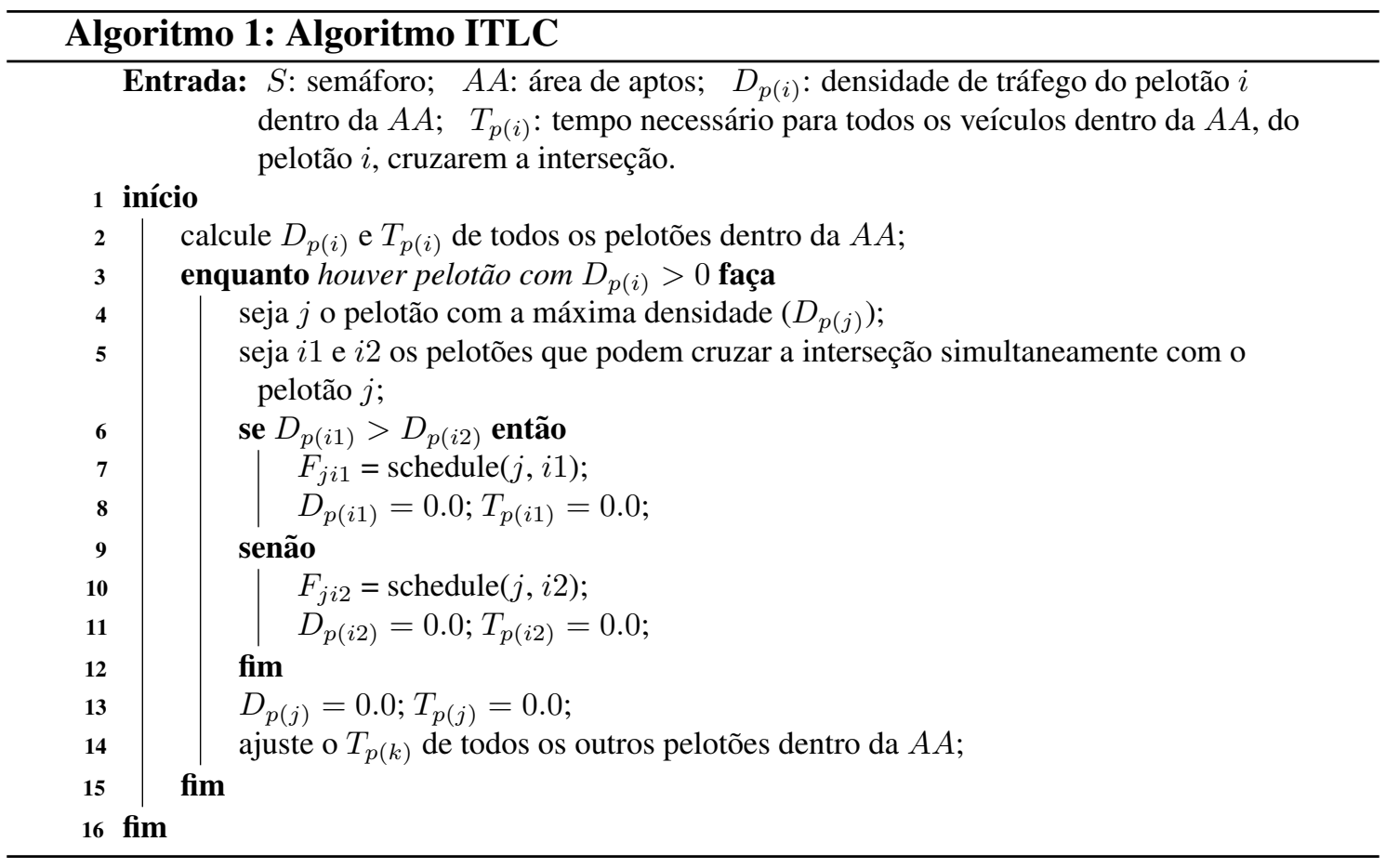

$$
T_{p(i)}=\min \left(\theta+\frac{D_{u v(i)}}{V_{p(i)}}, T_{\max }\right)
$$

Após a passagem de uma certa fase escalonada pela interseção, a densidade de tráfego $\left(D_{p(i)}\right)$ e o tempo estimado de viagem $\left(T_{p(i)}\right)$ de cada pelotão naquela fase são configurados para zero. Em seguida, o tempo estimado de viagem $T_{p(k)}$ para cada pelotão $k$ dos fluxos subsequentes são atualizados (linha 14). Após essas atualizações, o algoritmo repete o procedimento de escalonamento para todos os pelotões remanescentes de densidade não nula até todos serem escalonados.

A área de aptos do ITLC possui limites definidos pela média da velocidade de todos os fluxos conflitantes. Isso pode gerar pelotões que não sejam do tamanho mais apropriado para sua velocidade de fluxo. Pelotões com tamanho menor do que deveriam podem deixar de fora veículos que poderiam passar pela interseção. Por outro lado, pelotões com tamanho maior do que deveriam podem incluir áreas sem veículos que influenciem negativamente no cálculo das densidades dos mesmos. Além disso, é possível que o escalonamento de fases trabalhe com pelotões de densidades desatualizadas, visto que durante o ciclo não há qualquer atualização das densidades de pelotões não escalonados.

Por fim, a proposta em [Younes et al. 2016] tem como objetivo reduzir o atraso de veículos de emergência (corpo de bombeiros ou resgate, viaturas policiais e ambulâncias) nas interseções com semáforos. A solução, chamada CA-TLS (Context-Aware Traffic Light Self-Scheduling), é baseada no ITLC e reprograma as fases do semáforo quando um veículo de emergência se aproxima da interseção. Uma escala de prioridades também é definida para os casos onde há dois ou mais veículos de emergência. 


\section{Controle Adaptativo de Semáforo com Pelotões Veiculares Mais Acurados}

Esta seção apresenta a reformulação do algoritmo ITLC [Younes and Boukerche 2016] proposta neste trabalho. O algoritmo reformulado é chamado de ITLC-EP (Intelligent Traffic Light Controlling - Enhanced Platoons).

\section{1. Área Virtual de Aptos}

A Figura 1 ilustra um exemplo de área de aptos $(A A)$ em uma interseção com semáforo para o ITLC-EP. Seus limites podem ser diferentes em cada uma das aproximações.

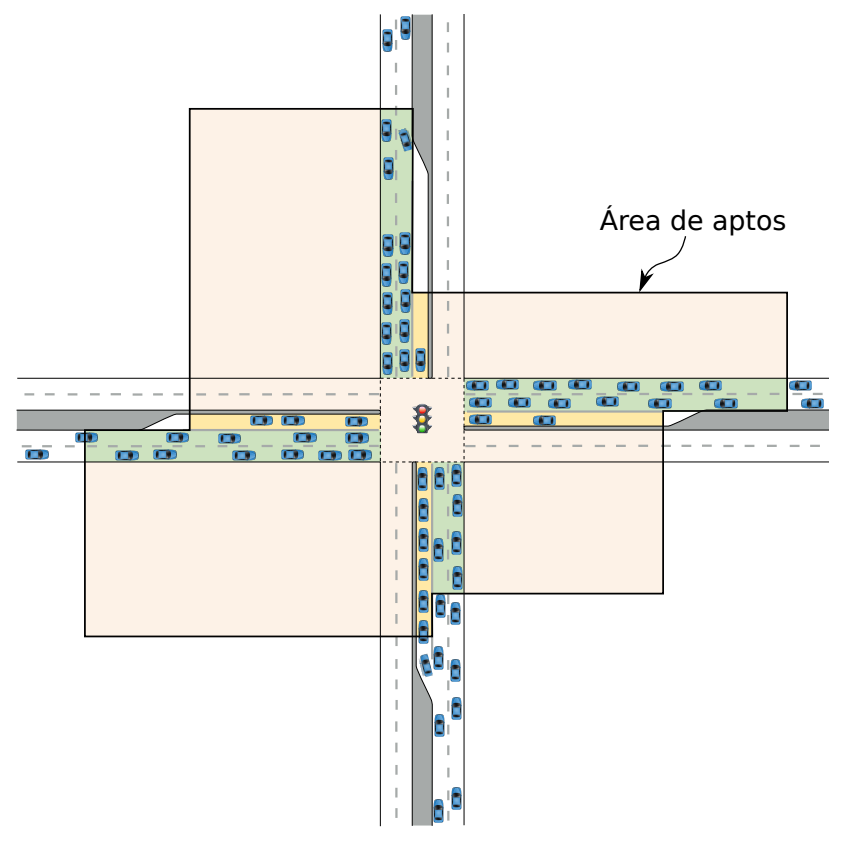

Figura 1. Área virtual de aptos para o ITLC-EP.

Conforme define a Equação 3, a distância entre o semáforo e o limite da área de aptos $D_{a a(i)}$, para cada fluxo $i$, é determinada com base na velocidade média do fluxo $i$ $\left(V_{f t(i)}\right)$ e o tempo de verde máximo $T_{\max (i)}$, permitido para o grupo focal desse fluxo $i$. Se $V_{f t(i)}$ for nula em algum instante, isso significa que todos os veículos do fluxo $i$ estão parados ou que nenhum veículo foi detectado no fluxo $i$ e, neste caso, $D_{a a(i)}$ permanece com o último valor calculado anteriormente. Em outras palavras, não haverá alteração no limite da área de aptos para aquele fluxo $i$.

$$
D_{a a(i)}=V_{f t(i)} \times T_{\max (i)}
$$

O período de tempo $T_{p(i)}$, necessário para todos os veículos dentro da $A A$, do pelotão $i$, cruzarem a interseção, não deve exceder o limite de tempo de verde máximo $T_{\max (i)}$ do grupo focal do fluxo $i$ onde o pelotão se encontra. Em outras palavras, todos os veículos localizados dentro da área de aptos, durante a fase de coleta de dados, devem ser capazes de atravessar a interseção, durante o tempo de verde máximo do semáforo para o fluxo correspondente. O valor de $T_{p(i)}$ é calculado pela Equação 4: onde $\theta, D_{u v(i)}$ e $V_{p(i)}$ são como definidos no ITLC e $C_{a c}$ é o comprimento da área de conflito (distância a ser percorrida pelos veículos na passagem pela interseção). A função min é responsável por limitar a $T_{\max (i)}$ o valor máximo do tempo para a fase de verde. 


$$
T_{p(i)}=\min \left(\theta+\frac{D_{u v(i)}+C_{a c}}{V_{p(i)}}, T_{\max (i)}\right)
$$

No algoritmo ITLC, os limites da área de aptos possuem a mesma distância para o semáforo em todos os fluxos. O tempo máximo de verde é o mesmo para todos os fluxos e a velocidade, utilizada para cálculo desses limites, é a média das velocidades de todos os fluxos conflitantes. Isso resulta uma área virtual de aptos que pode truncar pelotões que poderiam ser maiores ou estender pelotões que não precisariam ser tão grandes. Por outro lado, o ITLC-EP permite uma formação de pelotões mais acurada. Isso porque os limites da área virtual de aptos são definidos de forma independente, a partir da velocidade e do tempo máximo de verde de cada fluxo.

\subsection{Algoritmo de Escalonamento ITLC-EP}

No cruzamento mostrado na Figura 2, estão disponíveis oito pares de fluxos que podem passar simultaneamente pela interseção : $F_{15}, F_{25}, F_{16}, F_{26}, F_{37}, F_{47}, F_{38}$ e $F_{48}$. Apenas quatro dessas oito fases precisam ser selecionadas em cada ciclo do semáforo. Por exemplo, se as fases $F_{15}$ e $F_{26}$ são escalonadas para atravessar, não será necessário escalonar as fases $F_{25}$ e $F_{16}$ durante aquele mesmo ciclo do semáforo porque os veículos nessas fases já foram escalonados por meio das fases escolhidas (isto é, $F_{15}$ e $F_{26}$ ).

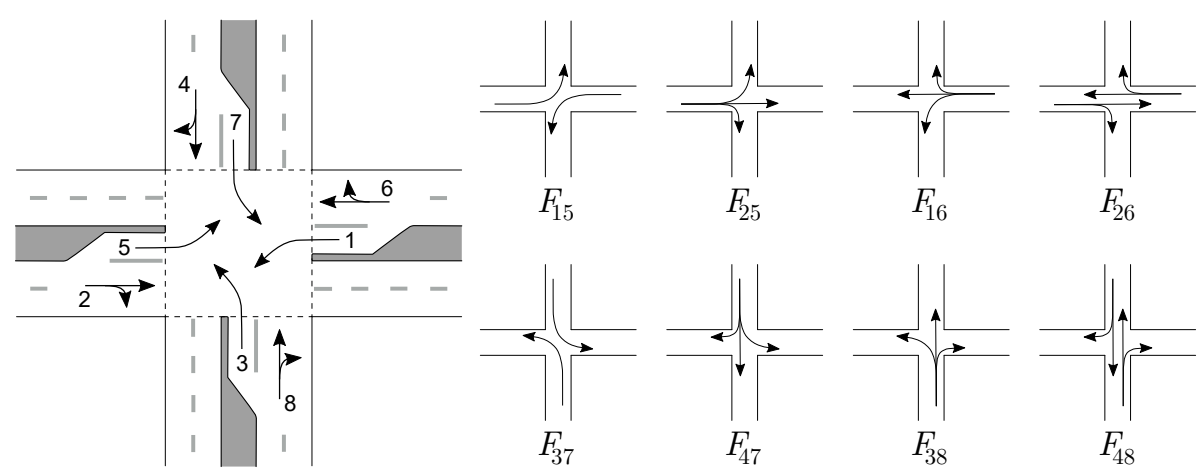

Figura 2. Interseção com 4 aproximações e suas 8 fases semafóricas possíveis.

O ITLC-EP escalona os pelotões dos fluxos conflitantes começando pelo de maior densidade, como mostra o Algoritmo 2. Inicialmente, as densidades e os tempos estimados de viagem de todos os pelotões são calculados e marcados como pelotões não escalonados (linhas 2-3). Dois fluxos de tráfego são candidatos a passar simultaneamente com o pelotão de mais alta densidade (linhas 5-6). Por exemplo, se o fluxo de tráfego 1 tem o pelotão de maior densidade, então os pelotões dos fluxos 5 ou 6 são elegíveis para passar com fluxo 1 (isto é, $F_{15}$ e $F_{16}$ ). O fluxo de tráfego que tiver o pelotão de maior densidade entre esses dois fluxos elegíveis é selecionado para passar na mesma fase com o pelotão de mais alta densidade (isto é, 1).

Em seguida, assim que dois fluxos de tráfego são incluídos em uma fase, o maior tempo necessário $\left(T_{p(i)}\right)$ é selecionado para a respectiva fase escalonada (linha 8 ou 12). Isso porque o tempo de cada fase é determinado pelo veículo mais distante dentro da área de aptos de cada fluxo de tráfego na fase selecionada, ou seja, o último veículo do pelotão. 


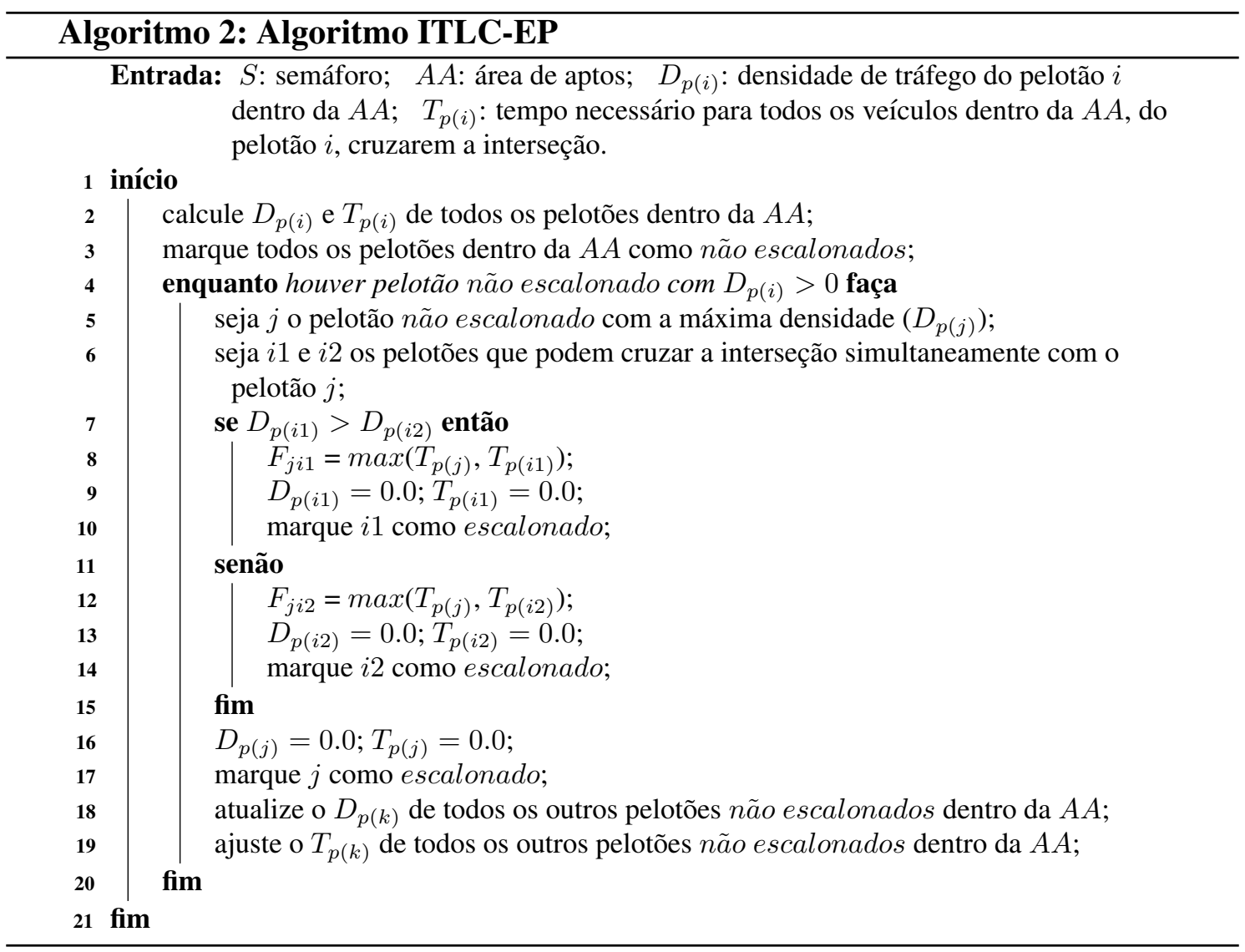

Depois que uma certa fase escalonada passa pela interseção, a densidade de tráfego $\left(D_{p(i)}\right)$ e o tempo necessário para todos os veículos dentro da $A A$ cruzarem a interseção $\left(T_{p(i)}\right)$ de cada pelotão naquela fase são configurados para zero e, então, o pelotão é marcado como escalonado. Após isso, a densidade $D_{p(k)}$ e o tempo estimado de viagem $T_{p(k)}$ para cada pelotão $k$ dos fluxos subsequentes são atualizados (linhas 1819). Os valores de $D_{p(k)}$ são atualizados porque, durante a passagem pelo semáforo da fase anteriormente escalonada, é possível que novos veículos tenham entrado na área de aptos provocando uma alteração na ordem das densidades dos pelotões remanescentes. Já a atualização dos valores de $T_{p(k)}$ visa ajustá-los de acordo com a nova localização e velocidade estimada para o último veículo em cada pelotão.

Após essas atualizações, o algoritmo repete o procedimento de escalonamento para todos os pelotões remanescentes de densidade não nula até todos serem escalonados. Pelotões vazios são eliminados do escalonamento atribuindo zero ao valor de suas densidades. Quando todos os pelotões de densidade não nula são marcados como escalonados (isto é, quando todos os veículos, detectados dentro da área de aptos, passam pela interseção com sucesso), uma nova etapa de avaliação de tráfego dentro da área de aptos é realizada e o algoritmo de escalonamento de fases é executado repetidamente para os veículos recém chegados.

No ITLC as densidades dos pelotões são calculadas apenas no início de um ciclo semafórico. Já no ITLC-EP, adicionalmente, há a atualização das densidades dos pelotões remanescentes no decorrer do ciclo, após o escalonamento de cada fase. Em um tempo de 
ciclo inteiro, alguns novos veículos podem entrar na área de aptos e comprometer os valores calculados anteriormente no início do ciclo. Por exemplo, um pelotão remanescente que não era o de maior densidade, no cálculo inicial pré-ciclo, pode ter recebido mais veículos e se tornado o de maior densidade durante o tempo de ciclo. E sem a atualização que o ITLC-EP realiza, ele não seria escalonado de forma prioritária.

A forma que o ITLC-EP utiliza para obter as características de tráfego de fluxos conflitantes é a mesma utilizada pelo ITLC, o protocolo ECODE. Esse fato é importante para assegurar uma comparação justa na avaliação de desempenho dos dois algoritmos.

\section{Avaliação de Desempenho}

A avaliação do ITLC-EP foi realizada por meio de simulações computacionais em dois cenários: um sintético e um realístico. Os resultados obtidos são comparados aos controles semafóricos de tempo fixo e ITLC. As principais métricas avaliadas são: (i) vazão de veículos no semáforo, (ii) atraso médio dos veículos no semáforo, (iii) emissão média de $\mathrm{CO}_{2}$ por veículo (calculada por meio do modelo EMIT (EMIssions from Traffic [Cappiello et al. 2002]) e (iv) overhead de sinalização. No trabalho que especifica o algoritmo ITLC apenas as duas primeiras métricas são avaliadas em um cenário sintético.

A duração de cada simulação realizada não possui um tempo fixo preestabelecido. O final da simulação ocorre no momento em que o último veículo chega ao seu destino. As vias possuem limite de velocidade máxima de $50 \mathrm{Km} / \mathrm{h}$ e as conversões à direita não são livres (são permitidas apenas ao sinal verde do semáforo). Cada experimento foi replicado 30 vezes para cada uma das soluções avaliadas e o nível de confiança para cada experimento é $95 \%$.

Todos os tempos fixos ou limitantes das fases semafóricas foram definidos de acordo com o método de Webster [Webster and Cobbe 1966], seguindo as recomendações do CONTRAN (Conselho Nacional de Trânsito) [CONTRAN 2014].

A frequência de transmissão dos beacons é de $1 \mathrm{~Hz}$. Já a potência de transmissão dos veículos foi de 7,1 miliwatts $(\mathrm{mW})$, o que se traduz em um raio de comunicação de cerca de 300 metros. O modelo de propagação utilizado foi o Two-Ray Interference [Sommer and Dressler 2011].

Como ferramentas de simulação foram utilizados o simulador de rede $\mathrm{OMNeT}++$ 5.3 [Varga and Hornig 2008] e o simulador de tráfego e mobilidade urbana SUMO 0.32.0 [Krajzewicz et al. 2012]. O framework Veins 4.7.1 [Sommer et al. 2011], que possui suporte para o padrão IEEE 802.11 p, foi utilizado para integrar esses dois simuladores. Destaca-se que essa versão do Veins (4.7.1) implementa um módulo de semáforo que já traz consigo uma RSU integrada. Nas versões anteriores não havia essa integração.

\subsection{Semáforo em Cruzamento Sintético}

O cenário com semáforo em cruzamento sintético foi modelado no simulador SUMO sob uma região de aproximadamente $1 \mathrm{Km}^{2}$. A Figura 2 da Seção 3.2 representa este cenário. Cada aproximação do semáforo possui extensão de 500 metros e três faixas de trânsito. A faixa interna é exclusiva para veículos que vão converter à esquerda e se estende apenas até uma distância de 90 metros do semáforo. Este cenário é o mesmo utilizado em três dos trabalhos relacionados abordados anteriormente [Pandit et al. 2013] [Younes and Boukerche 2016] [Younes et al. 2016]. 
A demanda de tráfego gerada no cenário segue rotas com origem no início de uma dada aproximação e destino no final das outras aproximações. Na interseção, as probabilidades do veículo converter à esquerda, seguir em frente ou converter à direita são respectivamente 0,$15 ; 0,70$ e 0,15 . Essas probabilidades são as mesmas utilizadas no OAF [Pandit et al. 2013].

Foram realizados experimentos neste cenário com 5 demandas de tráfego diferentes. Nesses experimentos, a quantidade total de veículos destinados a passar pela interseção é de 200, 400, 600, 800 e 1000. Em cada demanda, o tráfego é distribuído igualmente entre cada uma das aproximações, ou seja, não há uma via com tráfego mais intenso que a outra. Cada fluxo gerado nas aproximações insere veículos na via de forma aleatória, seguindo uma probabilidade de 0,5 a cada segundo.

No controle de tempo fixo, as fases $F_{15}, F_{26}, F_{37}$ e $F_{48}$, mostradas na Figura 2, são utilizadas para compor o ciclo semafórico. Sendo de 10 segundos a duração das fases $F_{15}$ e $F_{37}$ e de 33 segundos das fases $F_{26}$ e $F_{48}$. Já para o semáforo operando com os algoritmos ITLC e ITLC-EP, as fases escalonadas onde os fluxos 2, 4, 6 e 8 estão presentes têm duração mínima de 10 segundos e máxima de 49,5 segundos. Enquanto para as fases escalonadas onde os fluxos $1,3,5$ e 7 estão presentes, a duração mínima é de 10 segundos e a máxima de 15 segundos.

Como mostra a Figura 3(a), em todas as demandas de tráfego o ITLC-EP obtém uma vazão de veículos no semáforo superior. Em relação ao ITLC, a vazão do ITLCEP é maior em todas as demandas de tráfego. Essa superioridade fica menos acentuada conforme a demanda de tráfego se torna maior. Com 200 veículos a vazão é 13,85\% maior e com a demanda de 1000 veículos o aumento da vazão é de 4,46\%. Isso ocorre porque as melhorias implementadas na formação e atualização dos pelotões são mais perceptíveis em tráfegos mais dinâmicos. Conforme a demanda de tráfego aumenta, as filas de veículos nas aproximações provocam uma redução nas velocidades médias dos fluxos. De acordo com a Equação 3, essa redução gera limites de área de aptos menores que englobam praticamente essas filas por inteiro. Com isso, as densidades dos pelotões não sofrem alterações significativas no decorrer do ciclo, isso por conta da baixa ou inexistente entrada de novos veículos na área de aptos praticamente lotada.

Conforme exposto na Figura 3(b), o ITLC-EP possui atraso médio dos veículos no semáforo menor que as outras duas soluções em todas as demandas de tráfego. Quando comparado ao ITLC, o atraso chega a ser 30,21\% menor no ITLC-EP com uma demanda de 200 veículos. Conforme a demanda de veículos aumenta, ainda se observa uma redução, porém menos acentuada. Com uma demanda de 1000 veículos a redução é de $12,50 \%$. Assim como no caso da avaliação da vazão, isso ocorre porque com o aumento da demanda de tráfego, as atualizações de pelotões durante o ciclo semafórico não são tão significativas.

O gráfico da Figura 3(c) mostra que o ITLC-EP consegue taxas de emissão média de $\mathrm{CO}_{2}$ por veículo menores que as outras soluções, em todas as demandas de tráfego. Com uma demanda de 1000 veículos, por exemplo, a taxa de emissão de $\mathrm{CO}_{2}$ com o ITLC-EP chega a ser 5,88\% menor que o ITLC. Isso ocorre devido ao aumento na taxa de vazão dos veículos e à redução no atraso médio dos veículos proporcionados pelo ITLCEP. Essas melhorias tornam o tráfego mais fluido e com menos variação na aceleração dos 
veículos o que contribui para menores taxas de emissão de $\mathrm{CO}_{2}$.

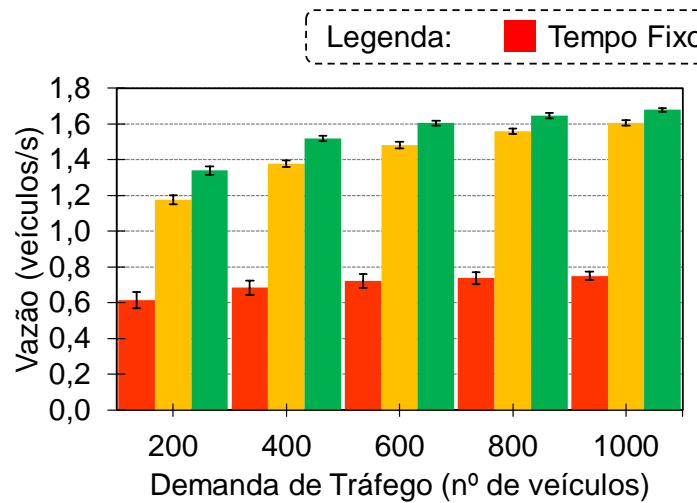

(a)

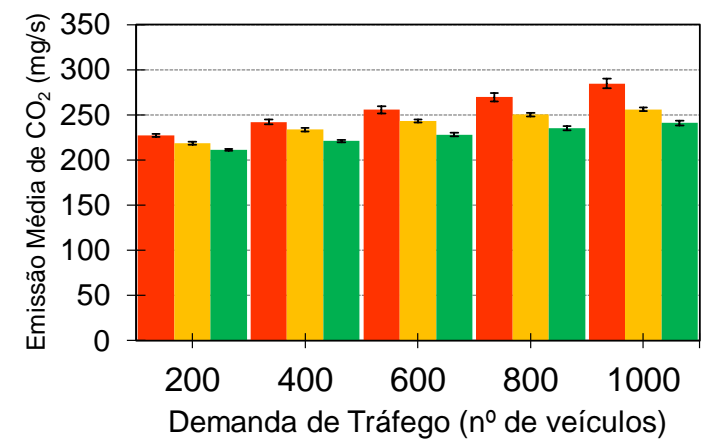

(c)

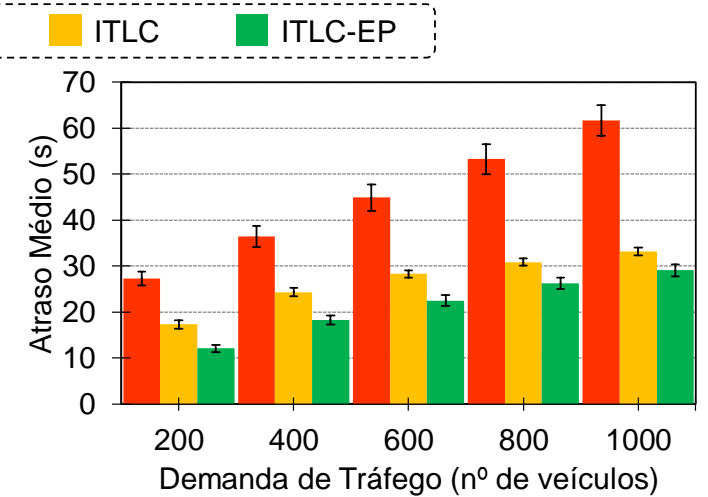

(b)

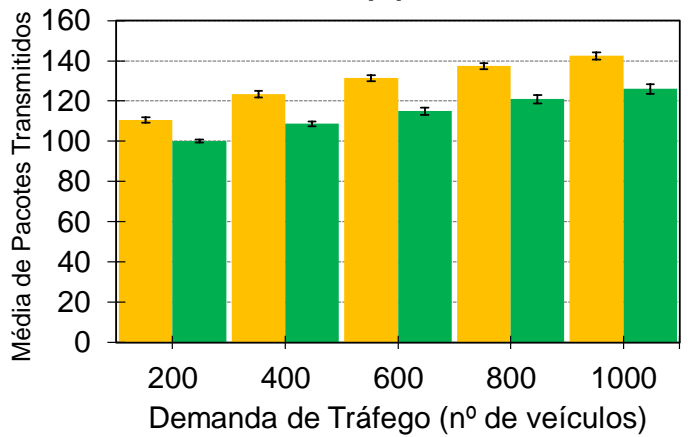

(d)

Figura 3. Métricas avaliadas no semáforo em cruzamento sintético. (a) Vazão de veículos no semáforo. (b) Atraso médio dos veículos no semáforo. (c) Emissão média de $\mathrm{CO}_{2}$ por veículo. (d) Overhead de sinalização.

A Figura 3(d) mostra que o ITLC-EP obtém menores taxas médias de transmissão de pacotes por veículo (overhead de sinalização) que o ITLC. Isso ocorre em todas as demandas de tráfego simuladas e a redução é, em média, 11,5\% menor em relação ao ITLC. A implementação do algoritmo ITLC-EP não difere do ITLC com respeito à quantidade e à frequência de envio de pacotes. No entanto, como os veículos que passam pela interseção, com o uso do ITLC-EP, levam menos tempo no cenário em virtude de uma maior vazão e de um menor atraso no semáforo, ocorre então que a quantidade de pacotes enviados por eles durante o percurso torna-se menor que na solução ITLC.

\subsection{Semáforo em Cruzamento Realístico na Cidade de Bolonha}

O cenário realístico da cidade de Bolonha na Itália foi desenvolvido por um grupo de pesquisa do projeto iTETRIS [Rondinone et al. 2013]. Ele compreende as áreas próximas à avenida Andrea Costa, que dá nome ao mesmo.

O cenário Andrea Costa possui uma demanda de tráfego referente a uma hora de trânsito intenso na cidade, mais precisamente, entre 8 e 9 horas da manhã. Essa demanda de tráfego foi mapeada por 636 equipamentos de detecção localizados em pontos espalhados pela cidade. Além disso, a prefeitura de Bolonha contribuiu informando sobre o posicionamento dos semáforos e seus planos semafóricos [Bieker-Walz et al. 2015].

Há 16 interseções com semáforos no cenário Andrea Costa. A interseção entre as avenidas Tolmino e Montefiorino, destacada na Figura 4(a), foi a escolhida para realização 
das simulações. Ela possui 8 fluxos de tráfego conflitantes com vias que dão passagem para aproximadamente 3600 veículos durante o tempo total de simulação disponível.

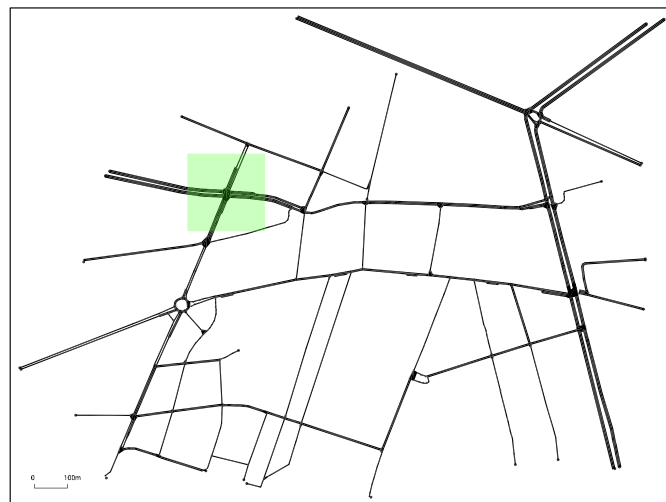

(a)

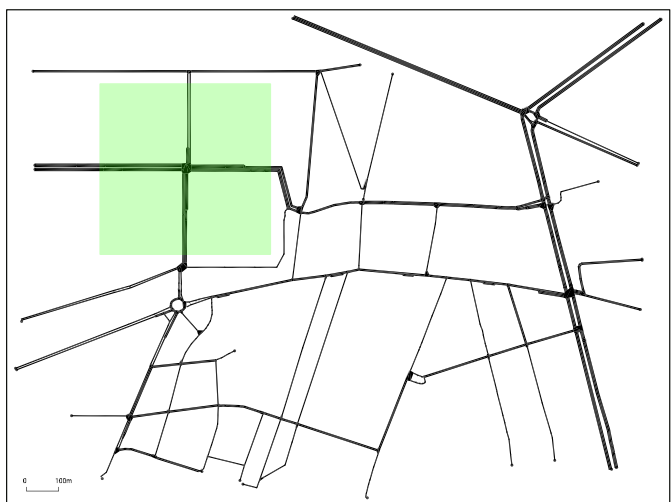

(b)

Figura 4. Cenário Andrea Costa e destaque da interseção com o semáforo utilizado nas simulações. (a) Semáforo com vias não adaptadas ao ECODE. (b) Semáforo com vias adaptadas ao ECODE.

A Figura 4(b) destaca como a interseção ficou após as adaptações necessárias para uso do ECODE como protocolo de detecção e quantificação do tráfego. Vale ressaltar que o ECODE foi concebido para áreas urbanas com layouts em grade [Younes and Boukerche 2015]. A área de $0,25 \mathrm{Km}^{2}$ destacada nessa Figura 4(b) também representa a região de interesse simulada.

Os 8 fluxos de tráfego da interseção podem ser combinados em 14 fases diferentes como mostrado na Figura 5. As fases $F_{145}^{\prime}, F_{245}^{\prime}$ e $F_{478}^{\prime}$ foram ignoradas nas simulações porque ambos os algoritmos ITLC e ITLC-EP não realizam o escalonamento de fases com mais de dois fluxos. Para manter a avaliação de desempenho justa, essas fases com três fluxos também foram desconsideradas no controle de tempo fixo.

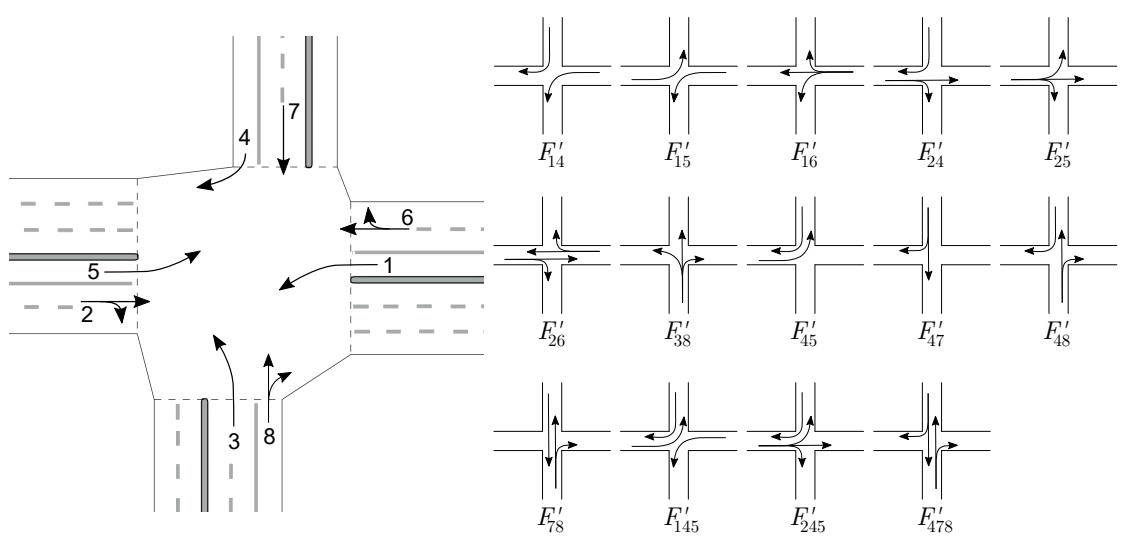

Figura 5. Interseção do cenário Andrea Costa e suas 14 fases possíveis.

No controle de tempo fixo do semáforo de Bolonha, as fases $F_{15}^{\prime}, F_{26}^{\prime}, F_{38}^{\prime}$ e $F_{47}^{\prime}$, mostradas na Figura 5, são utilizadas para compor o ciclo semafórico. Sendo de 10 segundos a duração da fase $F_{15}^{\prime}, 33$ segundos das fases $F_{26}^{\prime}$ e $F_{38}^{\prime}$ e 24 segundos da fase $F_{24}^{\prime}$. Já para o semáforo operando com os algoritmos ITLC e ITLC-EP, todas as fases escalonadas possuem duração mínima de 10 segundos. Para fases escalonadas onde os fluxos 1, 4, 5 
e 8 estão presentes a duração máxima é de 15 segundos. Para fases escalonadas onde os fluxos 2, 3 e 6 estão presentes a duração máxima é de 49,5 segundos. E para fases escalonadas onde o fluxo 7 está presente a duração máxima é 36 segundos. Em fases compostas por fluxos de duração máxima com tempos distintos, prevalece o maior tempo.

A Figura 6(a) apresenta a vazão de veículos no semáforo de Bolonha em cada uma das soluções. Observa-se que o ITLC-EP obtém uma vazão superior às outras. Em relação ao ITLC, a vazão do ITLC-EP é 3,25\% maior. Esse aumento está em conformidade com os resultados observados no cruzamento sintético. Com grandes demandas de tráfego, o ITLC-EP obtém vazões de veículos próximas às vazões do ITLC.

De acordo com o gráfico exposto na Figura 6(b), o ITLC-EP possui atraso médio dos veículos no semáforo de Bolonha menor que as outras duas soluções. Quando comparado ao ITLC, o atraso é 8,67\% menor no ITLC-EP. Assim como no caso da avaliação da vazão, mas de forma menos acentuada, grandes demandas de tráfego aproximam o comportamento do ITLC-EP ao do ITLC.

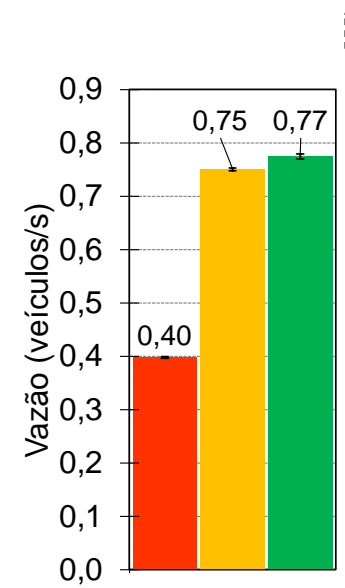

(a)

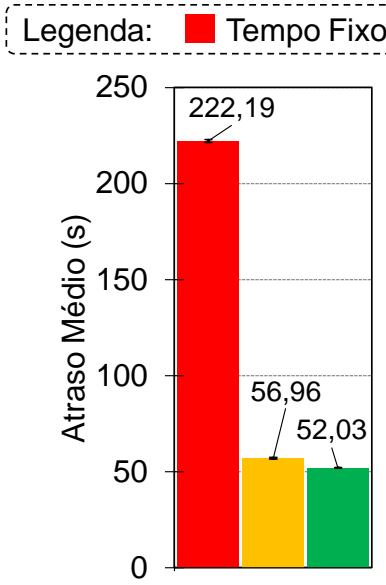

(b)

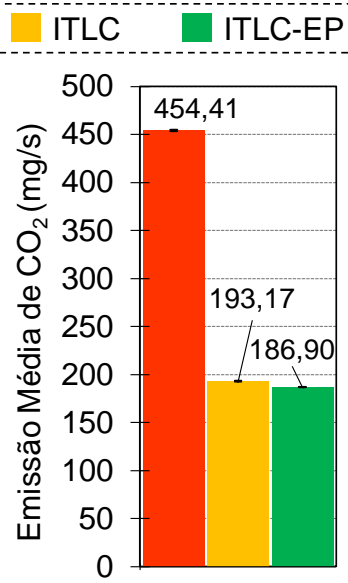

(c)

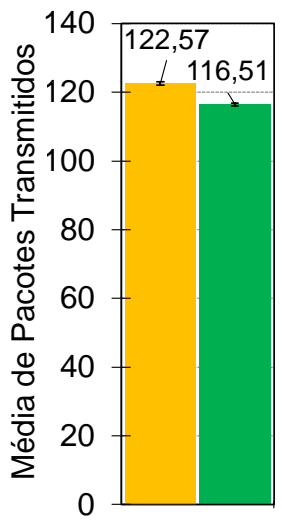

(d)

Figura 6. Métricas avaliadas no semáforo em cruzamento realístico na cidade de Bolonha. (a) Vazão de veículos no semáforo. (b) Atraso médio dos veículos no semáforo. (c) Emissão média de $\mathrm{CO}_{2}$ por veículo. (d) Overhead de sinalização.

Pela Figura 6(c), observa-se que o ITLC-EP obtém taxas de emissão média de $\mathrm{CO}_{2}$ por veículo no semáforo de Bolonha menores que as outras soluções. A taxa de emissão de $\mathrm{CO}_{2}$ com o ITLC-EP é 3,24\% menor que o ITLC. Assim como no cruzamento sintético, mais uma vez o aumento na taxa de vazão dos veículos e a redução no atraso médio dos mesmos são os responsáveis pela redução de emissão do poluente.

O gráfico da Figura 7 mostra a redução de emissão de $\mathrm{CO}_{2}$ (em toneladas) obtida pelo ITLC-EP em relação ao ITLC em um ano. Essa projeção considera 2 horários de pico em cada um dos 22 dias úteis no mês, em semáforos com as mesmas características do cenário realístico. Para absorver as 190 toneladas de $\mathrm{CO}_{2}$ emitidas na atmosfera com a operação de 16 semáforos seriam necessárias, por exemplo, mais de 27 mil árvores plantadas (considerando uma absorção de $7,0 \mathrm{Kg}$ de $\mathrm{CO}_{2}$ por árvore plantada por ano [Lacerda et al. 2009]).

O ITLC-EP obtém uma redução de 4,95\% em relação ao overhead de sinalização 


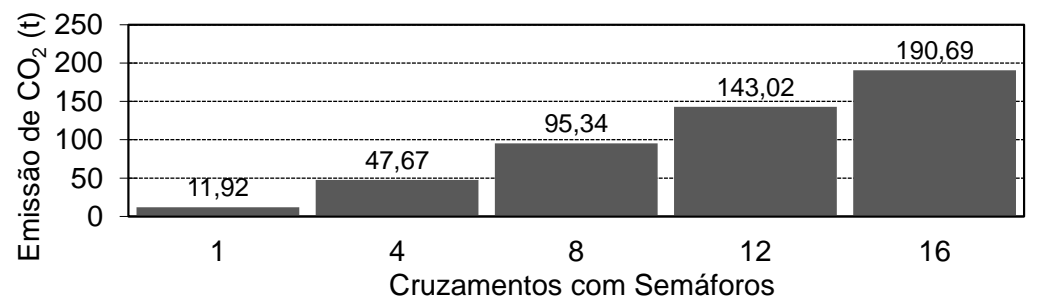

Figura 7. Redução de $\mathrm{CO}_{2}$ obtida pelo ITLC-EP em relação ao ITLC em 1 ano.

no semáforo de Bolonha obtido pelo ITLC. Esse resultado, mostrado na Figura 6(d), tem o mesmo significado já exposto na avaliação realizada no cruzamento sintético.

\section{Conclusões}

Neste trabalho foi proposto um algoritmo de controle adaptativo de semáforo com pelotões veiculares mais acurados chamado ITLC-EP. O ITLC-EP utiliza a tecnologia de redes veiculares e foi concebido a partir de melhorias introduzidas no ITLC [Younes and Boukerche 2016]. As melhorias modificaram as etapas de formação e atualização de pelotões veiculares.

$\mathrm{Na}$ avaliação do ITLC-EP frente aos controles ITLC e de tempo fixo, foram utilizados cenários com semáforo em cruzamento sintético e em cruzamento realístico na cidade de Bolonha na Itália. Em todos os cenários, o ITLC-EP aumentou a vazão de veículos no semáforo e reduziu o atraso médio dos veículos, a emissão de dióxido de carbono $\left(\mathrm{CO}_{2}\right)$ e o overhead de sinalização comparado aos outros dois controles semafóricos.

Conforme a demanda de tráfego aumenta, as melhorias introduzidas pelo ITLCEP ficam mais moderadas e a fluidez de tráfego obtida se aproxima mais dos resultados obtidos pelo ITLC. Contudo, vale ressaltar que nos centros urbanos a maior parte do tempo a situação é de tráfego mediano. Isso significa que na maior parte do tempo o ITLC-EP levaria uma vantagem considerável em relação ao ITLC, tendo ganho menos significativo apenas nos poucos horários de pico.

Como trabalhos futuros, pretende-se realizar um estudo do ITLC-EP atuando em cenários com quantidades críticas de veículos equipados com a tecnologia VANET. Também planeja-se adaptar o algoritmo ITLC-EP para trabalhar em vias arteriais com múltiplos semáforos coordenados entre si.

\section{Referências}

Bieker-Walz, L., Krajzewicz, D., Morra, A., Michelacci, C., and Cartolano, F. (2015). Traffic Simulation for All: A Real World Traffic Scenario from the City of Bologna. Lecture Notes in Control and Information Sciences, 13:47-60.

Booysen, M. J., Zeadally, S., and Van Rooyen, G. J. (2011). Survey of media access control protocols for vehicular ad hoc networks. IET Communications, 5(11):1619.

Cappiello, A., Chabini, I., Nam, E. K., Lue, A., and Zeid, M. A. (2002). A statistical model of vehicle emissions and fuel consumption. In Proceedings. The IEEE 5th International Conference on Intelligent Transportation Systems, pages 801-809.

CONTRAN (2014). Manual Brasileiro de Sinalização de Trânsito Volume V - Sinalização Semafórica. Conselho Nacional de Trânsito, V. 
Hsieh, T.-Y. and Tsai, P.-C. (2013). A novel traffic light management mechanism on intersection. In 2013 13th International Conference on ITS Telecommunications (ITST), pages $111-115$.

Krajzewicz, D., Erdmann, J., Behrisch, M., and Bieker Laura (2012). Recent Development and Applications of SUMO - Simulation of Urban MObility. International Journal On Advances in Systems and Measurements, 5(3-4):128-138.

Lacerda, J. S. D., Polizel, J. L., Couto, H. T. Z. D., Hiroto, M. M., and Pasishnyk, N. (2009). Estimativa da Biomassa e Carbono em Áreas Restauradas com Plantio de Essências Nativas. METRVM, 5:23.

Lee, W.-h., Lai, Y.-c., and Chen, P.-y. (2015). A Study on Energy Saving and $\mathrm{CO}_{2}$ Emission Reduction on Signal Countdown Extension by Vehicular Ad Hoc Networks. IEEE Transactions on Vehicular Technology, 64(3):890-900.

Pandit, K., Ghosal, D., Zhang, M., and Chuah, C.-N. (2013). Adaptive Traffic Signal Control With Vehicular Ad hoc Networks. IEEE Transactions on Vehicular Technology, 62(4):1459-1470.

Rondinone, M., Maneros, J., Krajzewicz, D., Bauza, R., Cataldi, P., Hrizi, F., Gozalvez, J., Kumar, V., Röckl, M., Lin, L., Lazaro, O., Leguay, J., Härri, J., Vaz, S., Lopez, Y., Sepulcre, M., Wetterwald, M., Blokpoel, R., and Cartolano, F. (2013). iTETRIS: A modular simulation platform for the large scale evaluation of cooperative ITS applications. Simulation Modelling Practice and Theory, 34:99-125.

Sommer, C. and Dressler, F. (2011). Using the Right Two-Ray Model? A Measurement based Evaluation of PHY Models in VANETs. In 17th ACM International Conference on Mobile Computing and Networking (MobiCom 2011), Poster Session, Las Vegas, NV. ACM.

Sommer, C., Eckhoff, D., German, R., and Dressler, F. (2011). A computationally Inexpensive Empirical Model of IEEE 802.11p Radio Shadowing in Urban Environments. 2011 8th International Conference on Wireless On-Demand Network Systems and Services, WONS 2011, pages 84-90.

Varga, A. and Hornig, R. (2008). An Overview of the OMNeT++ Simulation Environment. Proceedings of the 1st International Conference on Simulation Tools and Techniques for Communications, Networks and Systems \& Workshops, pages 60:1-60:10.

Webster, F. V. and Cobbe, B. M. (1966). Traffic signals. Road research technical paper. H.M.S.O.

Younes, M. B. and Boukerche, A. (2015). A performance evaluation of an efficient traffic congestion detection protocol (ECODE) for intelligent transportation systems. Ad Hoc Networks, 24(PA):317-336.

Younes, M. B. and Boukerche, A. (2016). Intelligent Traffic Light Controlling Algorithms Using Vehicular Networks. IEEE Transactions on Vehicular Technology, 65(8):58875899.

Younes, M. B., Boukerche, A., and Mammeri, A. (2016). Context-Aware traffic light selfscheduling algorithm for intelligent transportation systems. In 2016 IEEE Wireless Communications and Networking Conference, number Wcnc, pages 1-6. 